\title{
CD38 knockout natural killer cells expressing an affinity optimized CD38 chimeric antigen receptor successfully target acute myeloid leukemia with reduced effector cell fratricide
}

\section{Mark Gurney, ${ }^{1}$ Arwen Stikvoort, ${ }^{2}$ Emma Nolan, ${ }^{1}$ Lucy Kirkham-McCarthy, ${ }^{1}$ Stanislav Khoruzhenko, ${ }^{3}$ Rama Shivakumar, ${ }^{3}$ Sonja Zweegman, ${ }^{2}$ Niels W.C.J. van de Donk, ${ }^{2}$ Tuna Mutis, ${ }^{2}$ Eva Szegezdi, ${ }^{1}$ Subhashis Sarkar ${ }^{1 *}$ and Michael O'Dwyer ${ }^{1 *}$}

${ }^{1}$ National University of Ireland Galway, Galway, Ireland; ${ }^{2}$ Cancer Center Amsterdam, VU University Medical Center, Amsterdam, the Netherlands and ${ }^{3}$ MaxCyte, Inc., Gaithersburg, MD, USA

"SS and MOD contributed equally as co-senior authors.

\section{ABSTRACT}

T here is a strong biological rationale for the augmentation of allogeneic natural killer (NK) cell therapies with a chimeric antigen receptor (CAR) to enhance acute myeloid leukemia (AML) targeting. CD38 is an established immunotherapeutic target in multiple myeloma and under investigation as a target antigen in AML. CD38 expression on NK cells and its further induction during ex vivo NK cell expansion represent barriers to the development of a CD38 CAR-NK cell therapy. We set out to develop a CD38 CAR-NK cell therapy for AML, first by using an NK cell line which has low baseline CD38 expression and subsequently NK cells expanded from healthy donors. To overcome anticipated fratricide due to NK cell CD38 expression when using primary expanded NK cells, we applied CRISPR/Cas9 genome editing to disrupt the CD38 gene during expansion, achieving a mean knockdown efficiency of $84 \%$. The resulting CD38 knockdown expanded NK cells, after expression of an affinity optimized CD38 CAR, showed reduced NK-cell fratricide and an enhanced ability to target primary AML blasts. Furthermore, the cytotoxic potential of CD38 CAR-NK cells was augmented by pretreatment of the AML cells with all-trans retinoic acid which drove enhanced CD38 expression, offering a rational combination therapy. These findings support the further investigation of CD38 knockdown - CD38 CAR-NK cells as a viable immunotherapeutic approach to the treatment of AML.

\section{Introduction}

Acute myeloid leukemia (AML) is the most common acute leukemia in adults, accounting for approximately $2 \%$ of all cancer deaths. ${ }^{1}$ Curative treatment approaches remain chemotherapy-based, with allogeneic stem cell transplant consolidation for selected patients. The introduction of molecularly targeted therapies has provided important incremental improvements for specific AML subtypes. ${ }^{2-4}$ Relapsed disease, mediated by the persistence of chemotherapy-resistant leukemic stem cells (LSC) is particularly difficult to treat, and accounts for much of the mortality burden associated with AML. For many older patients, treatment options that are both tolerable and efficacious do not yet exist. Anti-CD19 chimeric antigen receptor (CAR) T-cell therapies have provided a ground-breaking approach to cancer immunotherapy in B-cell acute lymphoblastic leukemia and Bcell non-Hodgkin lymphomas., ${ }^{5,6}$ While there is considerable interest in applying the principle of CAR technology in other diseases, progress in AML has been limited to date by the absence of an ideal antigenic target, concerns about 'on-target off-tumor' toxicity including that to normal hematopoietic stem cells, and blast cell heterogeneity which exists both within and between patients. ${ }^{7-9}$.

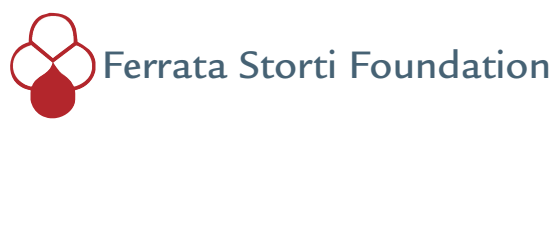

Haematologica 2022

Volume 107(2):437-445

\section{Correspondence:}

MICHAEL O'DWYER

michael.odwyer@nuigalway.ie

Received: September 11, 2020.

Accepted: December 22, 2020.

Pre-published: December 30, 2020.

https://doi.org/10.3324/haematol.2020.271908

(C)2022 Ferrata Storti Foundation

Material published in Haematologica is covered by copyright. All rights are reserved to the Ferrata Storti Foundation. Use of published material is allowed under the following terms and conditions:

https://creativecommons.org/licenses/by-nc/4.0/legalcode. Copies of published material are allowed for personal or internal use. Sharing published material for non-commercial purposes is subject to the following conditions:

https://creativecommons.org/licenses/by-nc/4.0/legalcode, sect. 3. Reproducing and sharing published material for commercial purposes is not allowed without permission in writing from the publisher. 
The multifunctional cell surface glycoprotein CD38, a breakthrough immunotherapeutic target in multiple myeloma, is also considered a potential target antigen in AML. In contrast to the uniformly high CD38 expression on malignant plasma cells, blast cell CD38 expression is heterogeneous although frequently exceeds that of normal cell populations. ${ }^{10}$ The CD38 monoclonal antibody daratumumab has been investigated in AML and has shown promising pre-clinical activity. ${ }^{10}$ CD38 CAR-T cells have been evaluated mainly for their activity in multiple myeloma and cytotoxicity against primary AML samples has also been confirmed. ${ }^{11}$ However, there remains concern about a potent myelosuppressive effect with a constituently expressed high-affinity anti-CD38 CAR due to CD38 expression on both mature myeloid cells and their precursors. ${ }^{11,12}$ To circumvent this problem, an affinity-optimized CD38 CAR has been developed to minimize the targeting of positive, but low-expressing normal cell populations. ${ }^{13}$

There is a strong biological rationale for natural killer (NK) cell-based approaches to adoptive cell transfer immunotherapy for AML. NK cells confer a component of the graft-versus-leukemia effect of allogeneic stem cell transplant and infusions of purified alloreactive NK cells have proven therapeutic potential. ${ }^{14-16}$ CAR-NK cell therapies are emerging as a complementary approach to CAR$T$ cells, with potential advantages including allogeneic cell sources and innate antigen independent anti-leukemic activity. An early clinical report of a cord-blood derived CD19 CAR-NK cell therapy has shown promising safety and efficacy in B-cell malignancies. ${ }^{17}$ We set out to develop and evaluate an affinity optimized CD38 CAR-NK cell therapy for AML. We first used the NK cell line KHYG-1, which has naturally low levels of CD38 expression. While allogeneic expanded NK (eNK) cell approaches are more suited to clinical translation, ex vivo NK cell expansion has been shown to lead to upregulation of CD38, which we also encountered using a feeder-free, interleukin-2-based expansion protocol. ${ }^{18}$ To reduce the anticipated NK cell fratricide that would occur using eNK cells, we applied CRISPR/Cas9 to disrupt the CD38 gene during NK cell expansion, creating fratricide-resistant NK cells prior to CD38 CAR expression. Both KHYG-1 and CD38 knockdown (KD) eNK approaches lead to efficient targeting of AML blasts upon CD38 CAR expression, with the degree of cytotoxicity correlating with CD38 expression. Finally, we confirm a rational combination approach utilizing all-trans retinoic acid (ATRA) to enhance CD38 expression on the AML cells. Collectively, our data support the potential of CD38 as a therapeutic target in AML and help to define a CD38 CAR-NK cell approach suited to clinical development.

\section{Methods}

\section{Ethical statement}

Healthy donor blood and AML patients' bone marrow samples were collected with written informed consent and approval from the institutional review boards at each institution (ref: CA2219). Cryopreserved samples were obtained from the biobank of Blood Cancer Network Ireland.

\section{Cells and reagents}

The cell lines THP-1, KG1a, U937 and KHYG-1 were obtained from the American Type Culture Collection and their identities confirmed by short tandem repeat profiling (Eurofins Genomics $^{\mathrm{TM}}$ ). CD38 CAR and mock KHYG-1 cells were generated by retroviral transduction with genomic integration confirmed by the inclusion of DsRed fluorescent protein. The development of the second-generation $\mathrm{CD} 28-\mathrm{CD} 3 \zeta$, optimized-affinity CD38 CAR was reported previously. ${ }^{13}$ Primary NK cells were isolated from healthy donor peripheral blood mononuclear cells after Ficoll-Paque density gradient centrifugation and negative immunomagnetic selection (NK Isolation Kit, Miltenyi Biotec ${ }^{\mathrm{TM}}$ ). NK cells were expanded in NK MACS medium (Miltenyi Biotec ${ }^{\mathrm{TM}}$ ) containing NK MACS supplement, $5 \%$ heat-inactivated human $A B$ serum and $100 \mathrm{U} / \mathrm{mL}$. interleukin-2 (PeproTech ${ }^{\mathrm{TM}}$ ). Cultures were pre-treated for $48 \mathrm{~h}$ with ATRA (Sigma-Aldrich ${ }^{\mathrm{TM}}$ ) or dimethyl sulfoxide, in the relevant experiments.

\section{CRISPR/Cas9 gene editing}

Five days after isolation, $5 \times 10^{5} \mathrm{NK}$ cells were electroporated with sgRNA-Cas9 complexes targeting multiple sites within the CD38 gene (Gene Knockout Kit V2, Synthego ${ }^{\mathrm{TM}}$ ) or control electroporated (MaxCyte ${ }^{\mathrm{TM}}$ GT flow transfection system). CD38-edited and control electroporated cells were expanded at a target density of $1 \times 10^{6}$ cells $/ \mathrm{mL}$. On day 13-15 of expansion, CD38 expression was assessed by flow cytometry. Knockdown efficiency was calculated as (\% CD38-positive cells [mock electroporated] - \% CD38-positive cells [CRISPR/Cas9 edited]).

\section{CD38 chimeric antigen receptor mRNA electroporation}

CD38 CAR mRNA was synthesized (Trilink Biotechnologies ${ }^{\mathrm{TM}}$ ) and $\mathrm{CD} 38 \mathrm{CAR}$ expression in primary CD38 KD and control eNK cells was achieved by electroporation $\left(100 \mu \mathrm{g} / \mathrm{mL}\right.$ mRNA, Maxcyte ${ }^{\mathrm{TM}}$ GT Flow Transfection System). CAR expression was confirmed by flow cytometry using anti-IgG $\mathrm{H}+\mathrm{L}$ specific goat anti-human antibody (JacksonImmuno research ${ }^{\mathrm{TM}}$ ) and biotinylated protein $\mathrm{L}$ stain (ACRO Biosystems $\left.{ }^{\mathrm{TM}}\right)$.

\section{Cytotoxicity assays}

Co-culture experiments involved 10,000 target cells (cell lines), or 20,000-50,000 bone marrow mononuclear cells from AML patients' samples. NK cell numbers were determined by the desired effector to target (E:T) cell ratio. After co-culture for 18-24 h, target cell lines or bone marrow mononuclear cells were identified by flow cytometry, using a cell-tracking dye: Tag-IT BV ${ }^{\mathrm{TM}}$ proliferation and cell tracking dye (Biolegend ${ }^{\mathrm{TM}}$ ) or VioletTrace $^{\mathrm{TM}}$ (Thermo Fisher). Primary blast cell populations were identified as CD45 ${ }^{\text {int }} / S_{S C} C^{\text {low }}$ (CD45 APC), supported by additional markers chosen based on clinical immunophenotyping data. Cell death was determined using propidium iodide (PI) or LIVE/DEAD Fixable Near-IR (Life Technologies L10119) staining and reported as '\% specific (blast) cytotoxicity' ([sample cytotoxicity - background cytotoxicity $] /[100$ - background cytotoxicity] x 100\%) or '\% blast cell cytotoxicity' as indicated.

\section{Statistical analysis}

GraphPad Prism 8 software (San Diego, CA, USA) was used for statistical analysis. Comparisons were conducted using multiple two-sided $t$-tests for cytotoxicity assays at each E:T ratio or one-way analysis of variance for cell expression data with statistical significance indicated by asterisks $\left({ }^{*} P<0.05,{ }^{* *} P<0.01\right.$, ${ }^{* * *} P<0.001$ and $\left.{ }^{* * * *} P<0.0001\right)$. Flow cytometry data were acquired on a BD FACS Canto II and analyzed using Flow Jo V10 software and Infinicyt (Cytognos ${ }^{\mathrm{TM}}$ ). 


\section{Results}

CD38 chimeric antigen receptor expression enhances KHYG-1 acute myeloid leukemia targeting

To assess the feasibility of targeting CD38 with a CARNK cell approach in AML, we first defined the CD38 expression profile of AML cell lines. We classified THP-1 and U937 as CD38-positive, and KG1a as CD38-negative for further experiments (Figure 1A). We also confirmed a low level of CD38 expression on the KHYG-1 cell line, previously shown to be a NK cell line with significant cytotoxic potential, expressing a high concentration of active perforin and signaling kinases. ${ }^{19}$ The low CD38 expression of KHYG-1 is in contrast to that of NK-92 cells, another NK cell line which has been investigated clinically as an adoptive cell therapy, but is strongly CD38-positive. ${ }^{18}$

CD38 CAR-KHYG-1 cells were generated by retroviral transduction using an 'affinity-optimized', second-generation anti-CD38 CAR (CD3\%-CD28). ${ }^{13}$ CD38 CARKHYG-1 cells displayed similar characteristics and kinetics in cell culture as those of mock-transduced KHYG-1
A

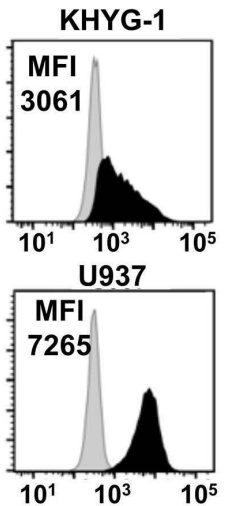

THP-1

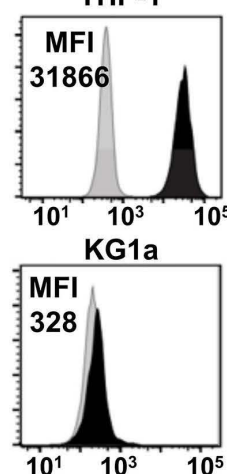

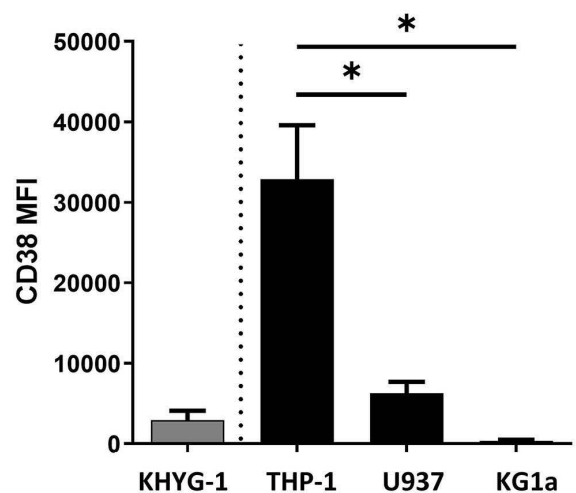

B

THP-1

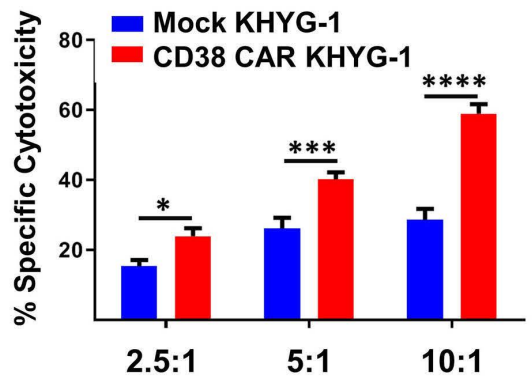

Effector : Target Ratio

U937

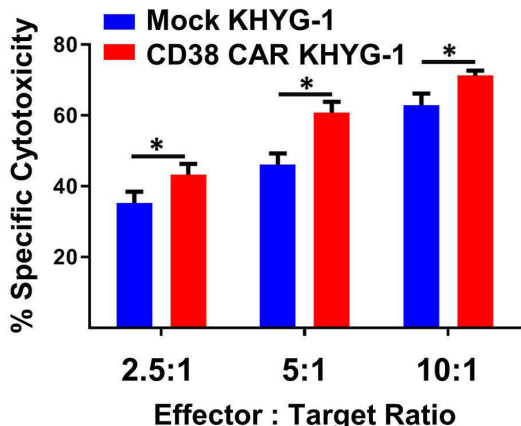

D
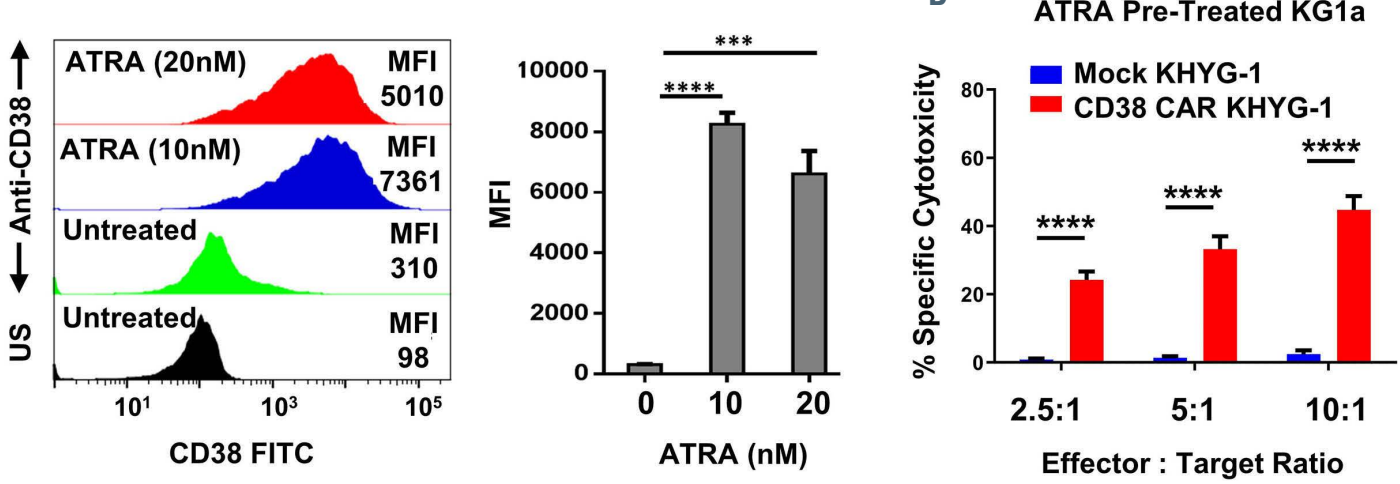

Figure 1. Affinity-optimized CD38 CAR-KHYG-1 cell cytotoxicity against acute myeloid leukemia cell lines. (A) Histograms depict CD38 expression of the KHYG-1 NK cell line and acute myeloid leukemia (AML) cell lines THP-1 (CD38-positive), U937 (CD38-positive) and KG1a (CD38-negative) with mean fluorescence intensity (MFI) indicated. Bars represent relative CD38 expression of KHYG-1 and AML cell lines ( $\mathrm{n}=3$ individual repetitions). Mean values for AML cell lines compared by an unpaired $t$-test. (B) Bar chart depicting the specific cytotoxicity of mock-transduced and CD38 CAR-KHYG-1 cells at varying effector to target (E:T) ratios against the CD38-positive cell lines THP1 and U937. Comparisons of four independent experiments made by an unpaired $t$-test at each effector to target (E:T) ratio. (C) Representative histograms depicting CD38 upregulation and MFI values in KG1a cells after $48 \mathrm{~h}$ of treatment with all-trans retinoic acid (ATRA) at $10 \mathrm{nM}$ and $20 \mathrm{nM}$ concentrations, compared to dimethylsulfoxide (DMSO) control treated KG1a cells. The bar chart summarize data from four independent experiments with comparisons by one-way analysis of variance. (D) Bar chart depicting specific cytotoxicity of mock-transduced and CD38 CAR-KHYG-1 cells in co-culture with $48 \mathrm{~h}, 10 \mathrm{nM}$ ATRA-pretreated KG1a cells at varying E:T ratios (summary of 4 experiments). Error bars indicate standard error of mean (SEM). Statistical significance is defined as $* P \leq 0.05, * * P \leq 0.01, * * * P \leq 0.001, * * * * P \leq 0.0001$. 
cells. To assess whether CD38 CAR targeting has an additional cytotoxic effect, we tested CD38 CAR-KHYG-1 cells and mock-transduced KHYG-1 cells against CD38positive AML cell lines (Figure 1B). CD38 CAR-KHYG-1 cells demonstrated greater cytotoxic effects against CD38-positive cell lines relative to mock-transduced KHYG-1 cells at all E:T ratios tested, with relatively greater increases seen against the strongly CD38-positive THP-1 cells (specific cytotoxicity $58 \%$ vs. $28 \%$ for THP1, 10:1 E:T ratio; $P<0.0001)$.

ATRA has been shown to upregulate CD38 expression across all AML subtypes, mediated by a retinoic acid response element in the first intron of the CD38 gene. ${ }^{20}$ Pretreatment with ATRA at $10 \mathrm{nM}$ for $48 \mathrm{~h}$ led to marked induction of CD38 expression on KG1a cells, which do not express detectable levels of CD38 in resting conditions (Figure 1C). CD38 CAR-KHYG-1 cells were cytotoxic to ATRA-pretreated KG1a cells, while mock-transduced KHYG-1 cells showed little cytotoxicity despite ATRA pretreatment (Figure 1D).

To better mimic the CD38 expression profile encountered in AML, we tested the efficacy of CD38 CARKHYG-1 cells against primary bone marrow mononuclear cells from AML patients (Figure 2A, B). CD38 CARKHYG-1 cells displayed greater specific cytotoxicity against AML blasts relative to mock-transduced KHYG-1 cells across a range of blast cell CD38 expression, with the degree of specific cytotoxicity correlating with blast cell CD38 expression (Figure 2C).

\section{CRISPR/Cas9 gene editing of CD38 in primary NK cells reduces NK cell fratricide upon CD38 chimeric antigen receptor expression}

While alloreactive NK cell approaches have shown some success in treating AML, we hypothesized that increased expression of CD38 during ex vivo NK cell expansion could be sufficient to trigger effector cell fratricide after expression of a CD38 CAR, despite affinity optimization. Indeed, we observed a consistent, mean 4fold increase in CD38 expression during feeder-free expansion of NK cells in interlueukin-2-containing media. Increases in CD38 from baseline (mean fluorescence intensity [MFI] 11,903) were detectable by day 5 (MFI 40,948) and persisted to at least day 13 (MFI 38,600) (Figure 3A). Extrapolating from our previous work on THP-1 cells (MFI 31,866), we concluded that this degree of CD38 expression would lead to a fratricidal effect upon CD38 CAR expression thus limiting the cytotoxic capacity of ex vivo-expanded CD38 CAR-NK cells.

We, therefore, set out to use CRISPR/Cas9 gene editing technology to disrupt the CD38 gene in primary NK cells. We used a multi-sgRNA format, introducing sgRNA-Cas9 complexes using a high-efficiency, electroporation-based approach on a platform scalable to Good Manufacturing Practice (GMP) grade development. CD38 KD and mock-electroporated cells were further expanded for use in functional assays. A consistent $\mathrm{KD}$ effect was achieved across all NK cell donors (mean $84 \%$; range, $75-92 \%$ ) (Figure 3B). CD38 KD was detectable 48 $\mathrm{h}$ after CRISPR/Cas9 gene editing, peaked by day 3-7 after electroporation and was stable across the duration of expansion suggesting minimal differences in the growth potential of CD38 KD and mock-electroporated NK cells in this expansion system (Online Supplementary Figure S1).
To confirm that CD38 $\mathrm{KD}$ eNK cells showed greater resistance to fratricide than wild-type eNK cells, we introduced mRNA coding for an affinity-optimized CD38 CAR. CAR expression was confirmed by complementary staining techniques - an anti-human IgG with light chain specificity, and biotinylated protein L, with control (background) and CAR staining depicted in Figure 3C. CD38 $\mathrm{KD}$ eNK cells displayed significantly less cell death than wild-type eNK cells, measured $18 \mathrm{~h}$ after CD38 CAR mRNA electroporation in the absence of target cells $(18 \%$ vs. $37 \%, P=0.002$ ) (Figure $3 \mathrm{D})$, confirming a greater resistance to fratricide. Furthermore, the biphasic CD38 expression pattern (representing the small residual CD38positive NK cell population after CRISPR/Cas9 gene editing) was lost in the $\mathrm{CD} 38 \mathrm{KD}$ population after $\mathrm{CD} 38$ CAR mRNA transfection, but not after non-specific (CD16) mRNA electroporation (Figure 3E). This emphasized the tendency of the CD38 CAR-NK cells to target CD38-positive eNK cells despite affinity-optimization of the CD38 CAR binding domain.

CD38 knockdown - CD38 chimeric antigen receptor-NK cells efficiently target primary acute myeloid leukemia blasts

To confirm that CD38 CAR expression in CD38 KD eNK cells enhances the activity of alloreactive NK cells against AML, CD38 KD eNK cells were electroporated with CD38 CAR mRNA or mock-electroporated prior to co-culture with bone marrow mononuclear cells from AML patients with a variety of molecular AML subtypes (Online Supplementary Table S1). CD38 KD - CD38 CARNK cells showed enhanced cytotoxicity relative to mockelectroporated CD38 KD cells, with the effect being most prominent at the highest E:T ratios tested (Figure 4A, B). Enhanced cytotoxicity was observed for all AML patients and cytotoxicity at the 5:1 E:T ratio correlated with blast cell CD38 expression $\left(\mathrm{R}^{2}=0.81\right)$ (Figure 4C).

We investigated the potential of ATRA pretreatment as a means of modulating CD38 expression and potentiating the effects of CD38 CAR targeting using CD38 KD CD38 CAR-NK cells. ATRA pretreatment induced a mean 5 -fold upregulation of surface CD38 expression in blast cells (Figure 4D). The increased CD38 expression was associated with greater sensitivity to CD38 KD - CD38 CAR-NK cells compared to dimethylsulfoxide-treated bone marrow mononuclear cell samples tested at the 2:1 and 5:1 E:T ratios (Figure 4E).

\section{Discussion}

We set out to augment the potential of NK cell adoptive transfer strategies in AML through expression of an affinity-optimized CD38 CAR. We demonstrated two potential approaches to CD38 CAR-NK cell therapy in this setting. We confirmed that CD38 KD eNK cells show reduced fratricide after CD38 CAR expression, allowing effective targeting of primary AML blasts. As an alternative approach we modified the NK cell line KHYG-1 to express a CD38 CAR, successfully targeting AML cell lines and primary samples. Both approaches could be enhanced by induction of CD38 expression using ATRA. We chose a NK cell line with naturally low CD38 expression to ensure viability after introducing a CD38 CAR. KHYG-1 cells have previously been shown to maintain 
cytotoxicity after irradiation and could be applied clinically in a similar manner to the NK-92 cell line. ${ }^{21}$ However, irradiation limits the potential for in vivo expansion and persistence - important variables in determining the clinical efficacy of cellular therapies. This requirement for irradiation may be avoided by using donor-derived, eNK cells, although this approach is further complicated by robust CD38 upregulation encountered during ex vivo expansion. Our CRISPR/Cas9 CD38 KD eNK cells reduce effector cell fratricide, representing an approach that could be explored clinically.

CD38 was a breakthrough immunotherapeutic target in multiple myeloma. While there is greater variability in CD38 expression in AML, CD38 is a potential target antigen in this disease. Daratumumab was shown to be active in an in vivo model of AML, while isatuximab has recently been examined in a large-scale, in vitro study. ${ }^{10,22}$
The expression pattern of CD38 in AML, in which there is often overlap with normal cell populations including myeloid and monocytic populations, raises concerns about considerable 'on-target, off-tumor' toxicity when a potent effector cell is directed toward CD38. High-affinity CD38 CAR strategies may maximize the proportion of patients for whom a CD38-directed therapy is likely to have activity, at the expense of considerable myelosuppressive effects. It is important to consider that not all offtumor effects are undesirable: in the case of CD38, elimination of CD38-positive immunoregulatory cell subsets may lead to a beneficial therapeutic effect. ${ }^{23,24}$ The efficacy of lower-affinity CD38 CAR strategies is likely to be limited to cases with strong expression or pharmacological upregulation of CD38. Herein we investigated an approach to CD38 CAR targeting in AML which aims to strike the balance of efficacy, applicability, and off-tumor
A

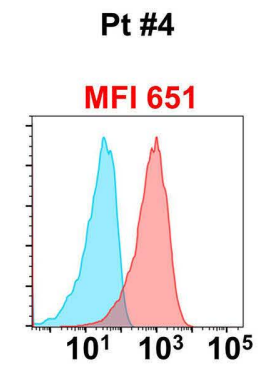

Pt \#6

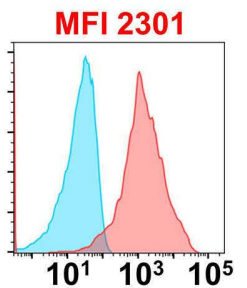

Pt \#7

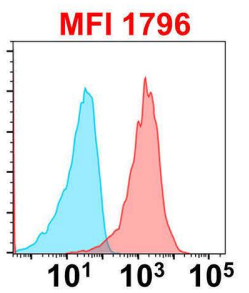

Pt \#8

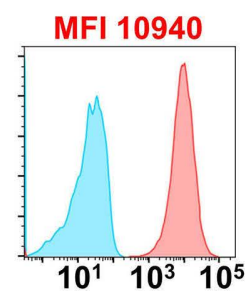

Pt \#9

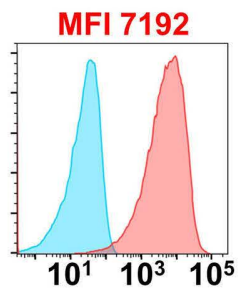

B

\section{MOCK - CD38 CAR}
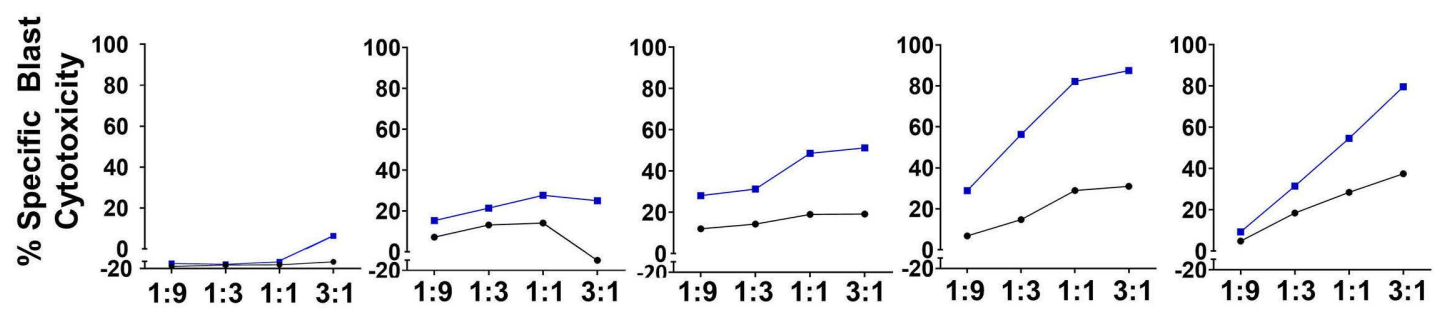

Effector : Target Ratio

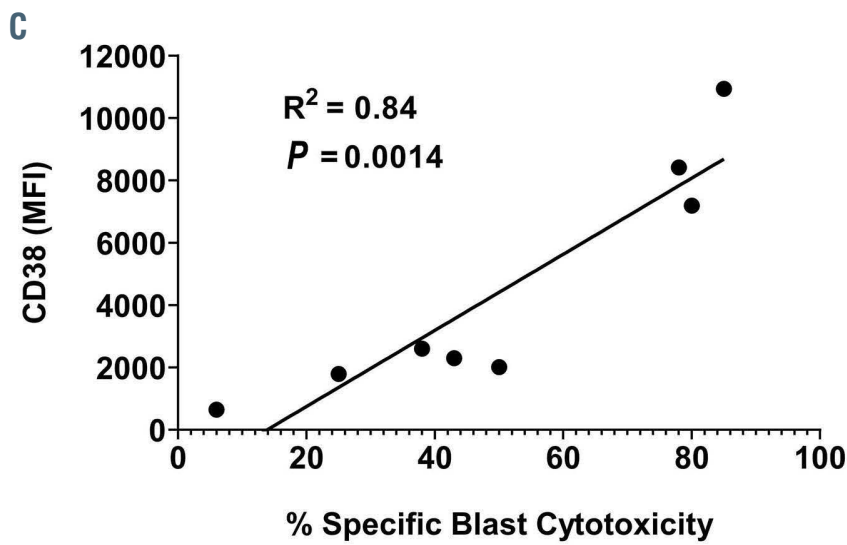

Figure 2. CD38 CAR-KHYG-1 activity against primary acute myeloid leukemia samples. (A) Histograms depict unstained controls (blue) and anti-CD38 stained blast cells (red), from a range of acute myeloid leukemia (AML) patients chosen to represent a spectrum of CD38 expression. Relative mean fluorescence intensity (MFI) figures for stained samples are reported. (B) Graphs represent specific blast cytotoxicity after co-culture assays with CD38 CAR transduced KHYG-1 (blue) and mocktransduced KHYG-1 (black) at specified effector to target (E:T) ratios for each corresponding patient's sample in Figure 2A. (C) The correlation plot and linear regression line depicts specific blast cell cytotoxicity at the E:T ratio of 3:1, versus CD38 expression (relative MFI) of primary AML samples from all co-culture experiments carried out in $2 \mathrm{~A}$, ( $\mathrm{n}=8$ experiments). 
effects. The established anti-leukemic activity of alloreactive NK cells in AML provides a rationale for the development of CAR-NK cell approaches. ${ }^{14,15}$ Alloreactive NK cells can be expected to retain their innate anti-leukemic activity, with enhancement against CD38-positive cells conferred by an anti-CD38 CAR. Toxicity against normal cell populations can be minimized through the use of an

A
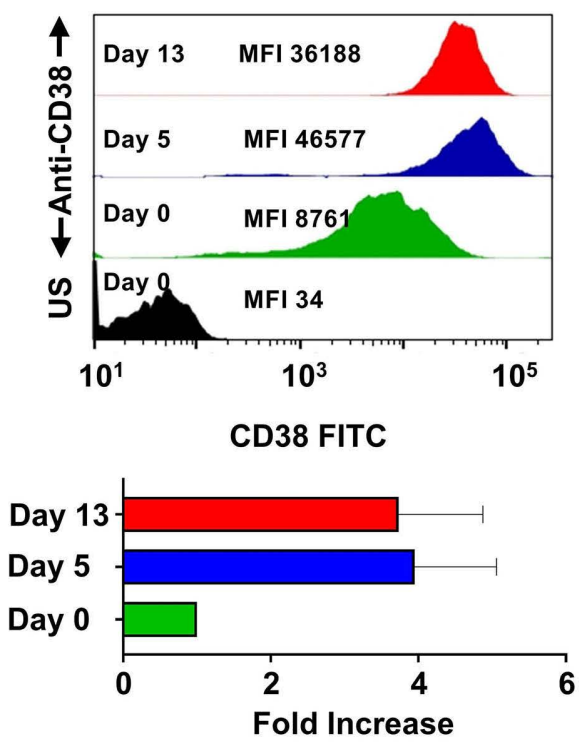

C Anti-Human IgG $\mathrm{H}+\mathrm{L}$ MOCK EP CD38 CAR MRNA EP
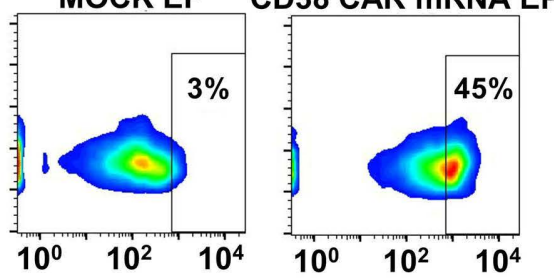

D

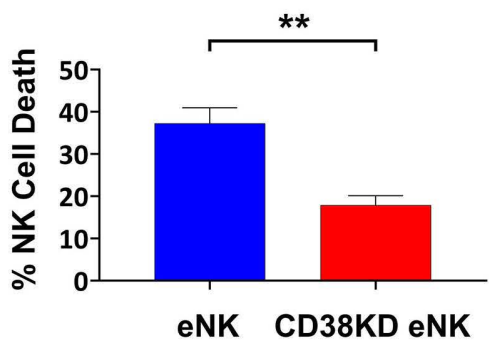

optimized-affinity CD38 CAR variant. ${ }^{11}$

While many target antigens are being considered in AML, CD38 is also unique in the availability of a licensed and well-tolerated oral agent capable of modulating target antigen expression, ATRA. ${ }^{20}$ Furthermore, it has been shown that malignant blast cells are particularly sensitive to the CD38-inducing effect of ATRA, acting directly
B
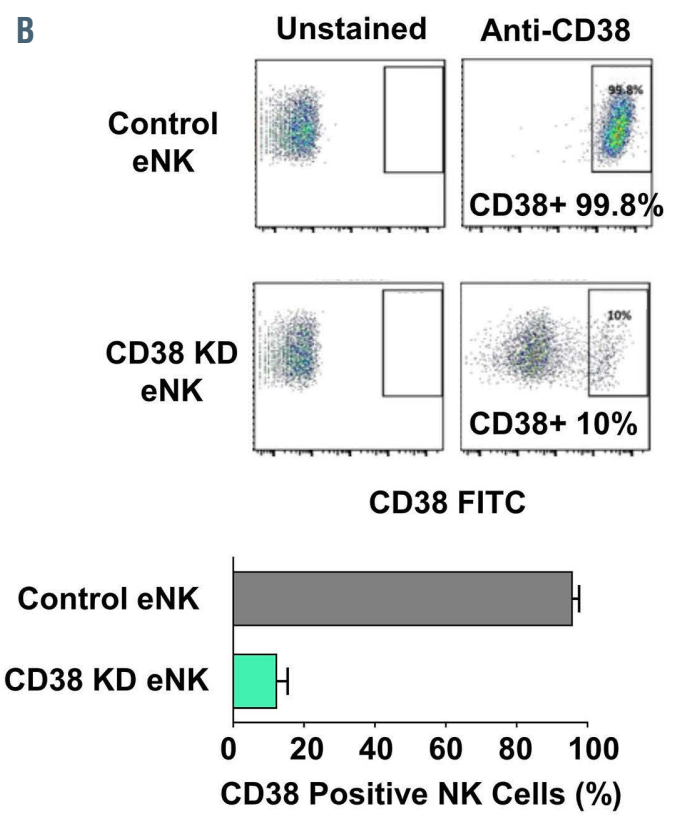

Biotinylated Protein L - Streptavidin

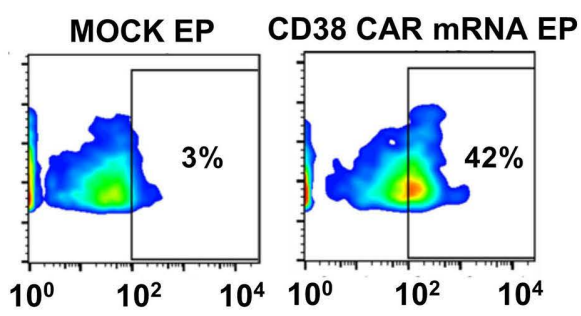

$\mathbf{E}$

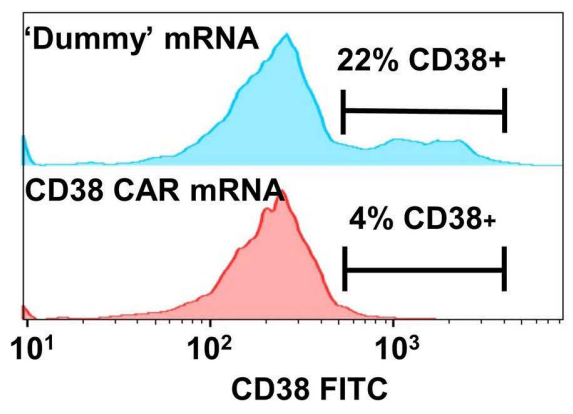

Figure 3. CRISPR/Cas9 gene editing of CD38 in primary expanded natural killer cells to reduce natural killer cell fratricide upon CD38 CAR expression. (A) CD38 expression in freshly isolated (day 0), and expanded natural killer (eNK) cells at day 5 and day 13, as measured by flow cytometry and presented as a representative histogram and summary bar chart of three unique expansions. (B) Residual CD38 expression measured on day 8-10 after CRISPR/Cas9 gene editing of CD38. Dot plots from representative donor for mock-electroporated and CD38 knockdown (KD) conditions. Bar chart represents summary data for four donors. (C) Confirmatory CAR staining performed $18 \mathrm{~h}$ after CD38 CAR mRNA electroporation. Pseudo-colored plots depict results from one representative experiment. (D) NK cell death after $18 \mathrm{~h}$ of culture after CD38 CAR mRNA electroporation comparing CD38 KD and control eNK cells across three individual experiments and NK cell donors. Comparison of mean cell death by an unpaired $t$-test. $(E)$ Histogram depicting a representative residual CD38 expression profile of viable CD38 KD eNK cells demonstrating loss of residual CD38-positive eNK cell population after CD38 CAR electroporation but not after 'dummy' mRNA (CD16) electroporation. EP: electroporated; $H+$ L: heavy and light chain specific. Error bars indicate standard error of mean (SEM). Statistical significance is defined as $* P \leq 0.05, * * P \leq 0.01$. 
through a retinoic acid response element within the CD38 gene. ${ }^{25}$ The vitamin $\mathrm{D}$ receptor agonist inecalcitol represents another investigational approach to CD38 modulation which could enhance the efficacy of CD38directed therapies in AML through a similar principle. ${ }^{26}$ The timing of a clinically applied combination therapy using ATRA and a CD38 CAR-NK cell would require careful planning. Evidence suggests that NK cell exposure to ATRA may have a net inhibitory effect on NK cell function, suggesting that the preferred approach may be ATRA prior to adoptive cell transfer. ${ }^{27}$

CD38 targeted therapies are complicated by NK cell CD38 expression, observed clinically with the NK celldepleting effects of daratumumab seen during the treat-
A 'Dummy'
mRNA EP

CD38 CAR EP
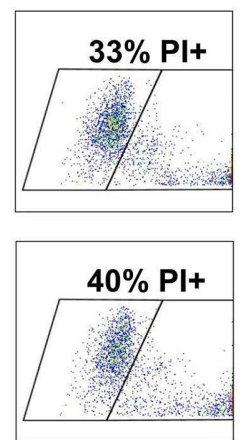

1:1
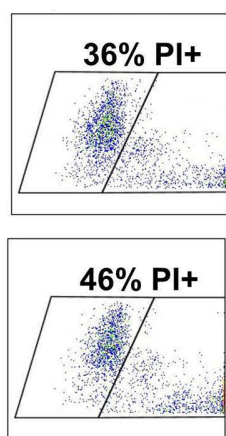

2.5:1
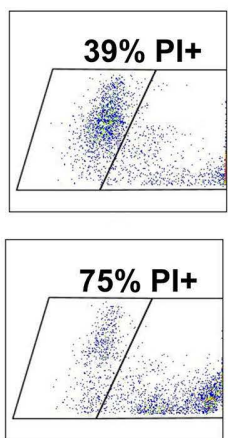

5:1

Effector : Target Ratio

B

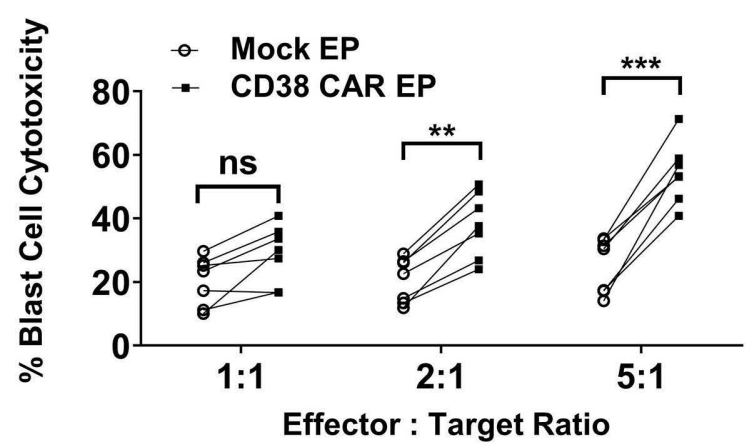

D

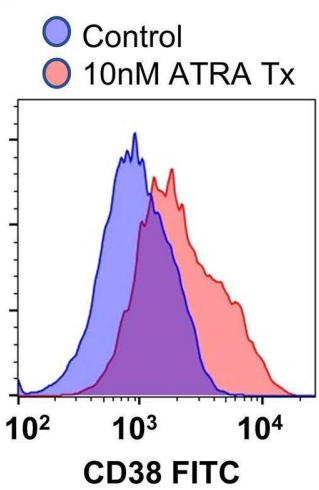

C

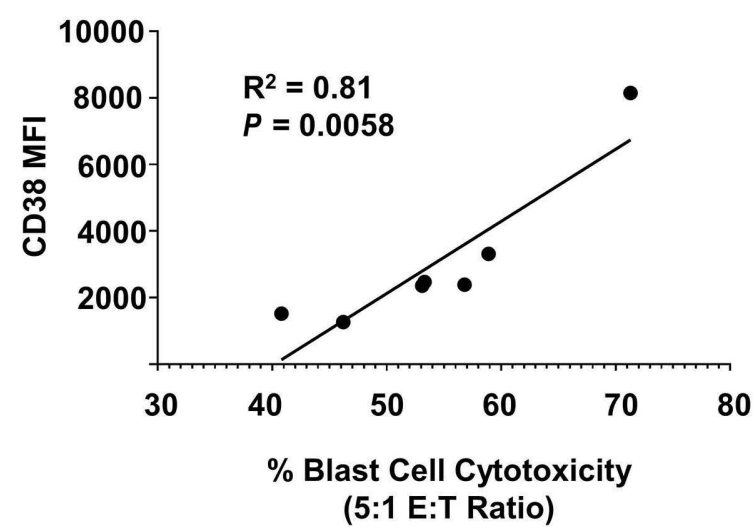

E
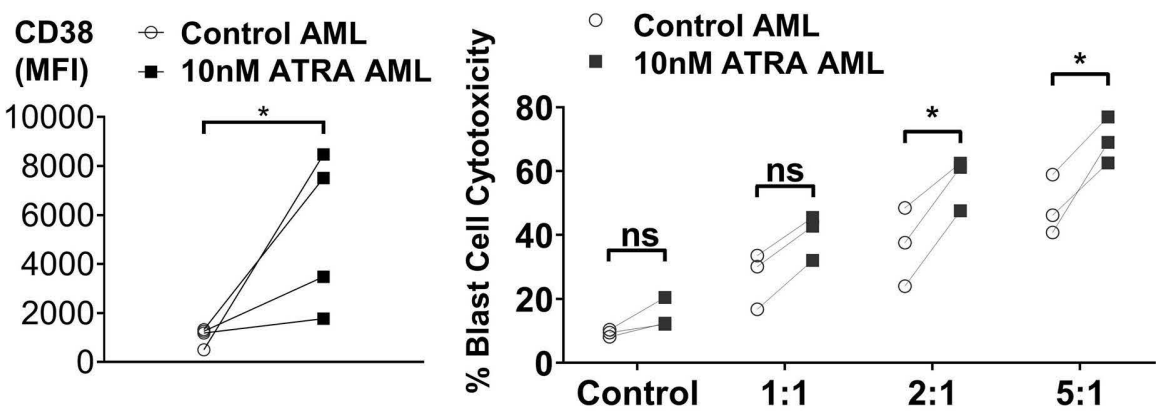

Figure 4. CD38 KD - CD38 CAR-NK cells show enhanced cytotoxicity against primary acute myeloid leukemia samples. (A) CD38 knockdown (KD) expanded natural killer (NK) cells were electroporated with CD38 CAR mRNA or 'dummy' mRNA (CD16) prior to co-culture with bone marrow mononuclear cells from acute myeloid leukemia (AML) patients. Blast cell cytotoxicity was measured by percentage of propidium iodide (PI)-positive cells (representative dot-plots are shown). (B) Summary data of co-culture assays as described in (A), for seven AML patients compared using unpaired t-tests for each effector to target (E:T) cell ratio. (C) The CD38 expression level of the blast population was correlated with the cytotoxic effect observed at an E:T ratio of 5:1 for experiments conducted in (B), and a linear regression model fitted using GraphPad Prism. (D) Bone marrow mononuclear cells from n=4 donors were treated with $10 \mathrm{nM}$ all-trans retinoic acid (ATRA) or dimethyl sulfoxide (DMSO) for $48 \mathrm{~h}$ prior to anti-CD38 staining. A representative histogram is displayed and summary data of four pooled donors were compared using an unpaired ttest. (E) ATRA or DMSO pretreated cells were co-cultured with CD38 KD-CD38 CAR-NK cells. Summary data from three experiments. Analysis by unpaired $t$-test for

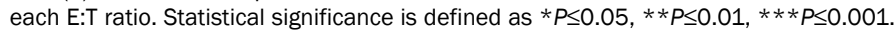


ment of multiple myeloma. ${ }^{28}$ Indeed, overcoming the fratricidal effect of daratumumab through combination with ex vivo eNK cells is actively under investigation in multiple myeloma. In keeping with prior reports, we observed CD38 upregulation during NK cell expansion, which was sufficient to lead to a fratricidal effect despite the use of an optimized-affinity CD38 CAR design. ${ }^{18}$ While it has been considered difficult to apply genetic engineering approaches to primary NK cells, we achieved a consistent, and high-efficiency disruption of the CD38 gene using a multi-sgRNA approach coupled with a flow transfection system. Our findings are comparable to recent descriptions of CRISPR/Cas9 editing in primary NK cells but using a different sgRNA design and expansion approach. ${ }^{27,29,30}$ The resulting CD38 KD eNK cells continued to expand and displayed reduced fratricide after CD38 CAR expression. With the availability of CRISPR/Cas9 and the relative ease of application to primary NK cells using clinically adaptable platforms now demonstrated by multiple groups, there are vast possibilities for this technology across NK cell therapeutics.

One potential limitation to CD38 targeting in AML is the limited capacity to target LSC populations, questioning the 'curative' potential of the therapies. LSC in AML are well-established, and while our understanding has evolved to include the existence of some CD38-positive LSC populations, it is likely that many LSC do reside within the traditional CD34-positive, CD38-negative compartment. ${ }^{31}$ Considering this feature of AML LSC, a CAR-NK cell targeting CD38 could be expected to have greater LSC targeting potential than a CAR-T cell, because of the presence of the innate activating pathways of NK cells above and beyond the CD38-specific CAR. Indeed the potential for long-term disease control, and thus LSC targeting capabilities can be inferred from data establishing the importance of NK cell KIR-ligand mismatch in the efficacy of allogeneic stem cell transplantation. ${ }^{16}$ Furthermore, a tandem CAR approach including a LSC-specific antigen and/or a variant of TRAIL (tumor necrosis factor related apoptosis inducing ligand) could be incorporated to augment LSC targeting. ${ }^{32}$ Tailored approaches using CAR modified NK cells targeting combinations based on the specific identified LSC immunophenotype in each case may ultimately be required given the absence of an identified universal LSC marker. This approach is becoming feasible with current and emerging technologies.

Antibody- and protein-based approaches have been considered previously in attempts to overcome fratricide in a CD38-directed CAR-T cell platform. ${ }^{33}$ CRISPR/Cas9generated, CD38 KD eNK cells have recently and successfully been applied to reducing the NK cell fratricidal effects of daratumumab with a focus on multiple myeloma. ${ }^{27}$ Interestingly, while a magnetic separation step was utilized to enhance the purity of the KD population in this innovative study, our data suggest that expression of a CD38 CAR combined with a highly efficient CRISPR/ Cas9 KD will likely lead to a self-selecting $\mathrm{KD}$ population without additional processing. While not the focus of our experiments, Kararoudi et al. ${ }^{27}$ also explored the cellular bioenergetic benefit of deletion of CD38 in eNK cells. $\mathrm{CD} 38$ converts nicotinamide adenine dinucleotide $\left(\mathrm{NAD}^{+}\right)$ to cyclic adenosine diphosphate-ribose through an enzymatic function. Additional $\mathrm{NAD}^{+}$availability due to loss of CD38 supplies an important co-factor favoring oxidative phosphorylation within NK cells. FT538, a NK cell product derived from induced pluripotent stem cells being developed by FATE Therapeutics, incorporates a CD38 deletion to overcome fratricide when combined with daratumumab. The group also demonstrated greater resistance to oxidative stress conferred by deletion of CD38, a characteristic likely to be favorable within the tumor microenvironment. ${ }^{34}$ These enhancements to NK cell biology suggest a broad range of applications for CD38 KD eNK cells beyond CD38 targeting and fratricide concerns. Simple and consistent approaches to their generation will likely be of clinical utility.

In conclusion, we present two viable approaches to CD38 CAR-NK cell therapies applied to AML. Both our CD38 CAR-KHYG-1 cells and CD38 KD eNK cell platforms overcome effector cell fratricide relating to NK cell CD38 expression. Furthermore, we report an efficient approach to CRISPR/Cas9 genome editing adapted to primary eNK cells and suitable for GMP expansion.

\section{Disclosures}

$M G$ has received educational funding from Janssen Pharmaceuticals and Takeda. AS and SS have received research funding from ONK Therapeutics Limited. LKM is an employee of ONK Therapeutics Limited. SK and RS are employees of Maxcyte Inc. SZ has received research funding from Takeda, Celgene, and Janssen and is a member of the board of directors or an advisory committee for Takeda, Celgene, and Janssen. NWCJvdD has received research funding from Janssen Pharmaceuticals, Amgen, Celgene, Novartis, and BMS and has participated in advisory boards for Janssen Pharmaceuticals, Amgen, Celgene, BMS, Takeda, Roche, Novartis, Bayer, and Servier. TM has received research funding from Gilead, Celgene, Novartis, ONK Therapeutics Limited, Genmab, Janssen and has been a member of an advisory board for Janssen. ES has collaborated in research projects with Janssen, Roche, Celgene, and Takeda. MOD has received research funding from ONK Therapeutics Limited, BMS, Celgene, and Glycomimetics; is a member of the board of directors or an advisory committee for Janssen, Abbvie, and ONK Therapeutics Limited; and owns equity in ONK Therapeutics Limited. EN has no potential conflicts of interest to disclose.

\section{Contributions}

SS and MOD conceived the research. EN, MG, AS and $L K M$ performed functional assays. SK and RS contributed to electroporation optimization. ES contributed to acquisition of patients' samples and the primary $A M L$ assay design. TM, $S Z$ and NVD developed CD38 CAR-KHYG1 cells and associated functional assays. $M G, S S$ and $A S$ wrote the manuscript and prepared the figures. All authors contributed to editing and reviewing the final manuscript prior to submission.

\section{Acknowledgments}

Reagents for electroporation were contributed by Maxcyte inc.

\section{Funding}

This research was supported by Irish Clinical Academic Training (ICAT) Programme fellowship funding to MG. ICAT is supported by the Wellcome Trust and the Health Research Board (grant number 203930/B/16/Z), the Health Service Executive National Doctors Training and Planning and the Health and Social Care, Research and Development Division, Northern Ireland. Work performed in collaboration with Blood Cancer Network Ireland was funded by Science Foundation Ireland and the Irish Cancer Society (Blood Cancer Network Ireland, 14/ICS/B3042). 


\section{References}

1. Surveillance, Epidemiology, and End Results (SEER) Program (1969-2018). U.S. Population Data with Other Software: (SEER Web site: www.seer.cancer.gov/popdata), National Cancer Institute, DCCPS, Surveillance Research Program, released December 2019

2. DiNardo CD, Stein EM, de Botton S, et al. Durable remissions with ivosidenib in IDH1-mutated relapsed or refractory AML. N Engl J Med. 2018;378(25):2386-2398.

3. Stein EM, DiNardo CD, Pollyea DA, et al. Enasidenib in mutant IDH2 relapsed or refractory acute myeloid leukemia. Blood. 2017;130(6):722-731.

4. Stone RM, Mandrekar SJ, Sanford BL, et al. Midostaurin plus chemotherapy for acute myeloid leukemia with a FLT3 mutation. N Engl J Med. 2017;377(5):454-464.

5. Maude SL, Laetsch TW, Buechner J, et al. Tisagenlecleucel in children and young adults with B-cell lymphoblastic leukemia. N Engl J Med. 2018;378(5):439-448.

6. Neelapu SS, Locke FL, Bartlett NL, et al. Axicabtagene ciloleucel CAR T-cell therapy in refractory large B-cell lymphoma. $\mathrm{N}$ Engl J Med. 2017;377(26):2531-2544

7. Cummins KD, Gill S. Chimeric antigen receptor T-cell therapy for acute myeloid leukemia: how close to reality? Haematologica. 2019;104(7):1302-1308.

8. Kenderian SS, Ruella M, Shestova O, et al. Targeting CLEC12A with chimeric antigen receptor $\mathrm{T}$ cells can overcome the chemotherapy refractoriness of leukemia stem cells. Biol Blood Marrow Transplant. 2017;23(3):S247-S248.

9. Petrov JC, Wada M, Pinz KG, et al. Compound CAR T-cells as a doublepronged approach for treating acute myeloid leukemia. Leukemia. 2018;32(6):1317-1326.

10. Naik J, Themeli M, de Jong-Korlaar R, et al. CD38 as a therapeutic target for adult acute myeloid leukemia and T-cell acute lymphoblastic leukemia. Haematologica. 2019; 104(3):e100-e103.

11. Drent E, Groen RWJ, Noort WA, et al. Preclinical evaluation of $\mathrm{CD} 38$ chimeric antigen receptor engineered $\mathrm{T}$ cells for the treatment of multiple myeloma. Haematologica. 2016;101(5):616-625

12. Mihara K, Yanagihara K, Takigahira M, et al. Activated T-cell-mediated immunotherapy with a chimeric receptor against CD38 in Bcell non-Hodgkin lymphoma. J Immunother.
2009;32(7):737-743

13. Drent E, Themeli M, Poels R, et al. A rational strategy for reducing on-target off-tumor effects of CD38-chimeric antigen receptors by affinity optimization. Mol Ther. 2017;25(8):1946-1958.

14. Björklund AT, Carlsten M, Sohlberg E, et al. Complete remission with reduction of highrisk clones following haploidentical NK-cell therapy against MDS and AML. Clin Cancer Res. 2018:24(8):1834-1844.

15. Nguyen R, Wu H, Pounds S, et al. A phase II clinical trial of adoptive transfer of haploidentical natural killer cells for consolidation therapy of pediatric acute myeloid leukemia. J Immunother Cancer. 2019;7 (1):81.

16. Ruggeri L, Capanni M, Urbani E, et al. Effectiveness of donor natural killer cell alloreactivity in mismatched hematopoietic transplants. Science. 2002;295(5562):20972100.

17. Liu E, Marin D, Banerjee P, et al. Use of CAR-transduced natural killer cells in CD19positive lymphoid tumors. $N$ Engl J Med. 2020;382(6):545-553.

18. Wang Y, Zhang Y, Hughes T, et al. Fratricide of NK cells in daratumumab therapy for multiple myeloma overcome by ex vivoexpanded autologous NK cells. Clin Cancer Res. 2018;24(16):4006-4023.

19. Suck G, Branch DR, Smyth MJ, et al. KHYG 1 , a model for the study of enhanced natural killer cell cytotoxicity. Exp Hematol. 2005;33(10):1160-1171.

20. Uruno A, Noguchi N, Matsuda K, et al. Alltrans retinoic acid and a novel synthetic retinoid tamibarotene (Am80) differentially regulate CD38 expression in human leukemia HL-60 cells: possible involvement of protein kinase C- $\delta$. J Leukoc Biol. 2011;90(2):235-247.

21. Tang X, Yang L, Li Z, et al. First-in-man clinical trial of CAR NK-92 cells: safety test of CD33-CAR NK-92 cells in patients with relapsed and refractory acute myeloid leukemia. Am J Cancer Res. 2018;8(6):10831089.

22.Zabaleta A, Tomas J, Simoes C, et al. The mode of action of the anti-CD38 monoclonal antibody (MAB) isatuximab in elderly acute myeloid leukaemia (AML). Hemasphere. 2020;4(S1):Abstract Book EP467.

23. Krejcik J, Casneuf T, Nijhof IS, et al. Daratumumab depletes CD38+ immune regulatory cells, promotes $\mathrm{T}$-cell expansion, and skews T-cell repertoire in multiple myeloma. Blood.2016;128(3):384-394.

24. Zhao C, Jia B, Wang M, et al. Multi-dimensional analysis identifies an immune signature predicting response to decitabine treatment in elderly patients with AML. Br J Haematol. 2020;188(5):674-684

25. Farber M, Arnold L, Chen Y, Möllmann M Duehrsen U, Hanoun M. Inhibition of CD38 shows anti-leukemic activity in acute myeloid leukemia. Blood. 2018;132(Suppl 1): $1456-1456$

26. Mouly E, Planquette C, Rousseau E, Delansorne R. Inecalcitol respectively induces or increases CD38 expression at the surface of CD38- or CD38+ AML cell lines representative of all $9 \mathrm{FAB}$ subtypes except M6. Cancer Res. 2018;78(13 Suppl):1890.

27. Naeimi Kararoudi M, Nagai Y, Elmas E, et al. CD38 deletion of human primary NK cells eliminates daratumumab-induced fratricide and boosts their effector activity. Blood. 2020;136(21):2416-2427.

28. Casneuf T, Xu XS, Adams HC, et al. Effects of daratumumab on natural killer cells and impact on clinical outcomes in relapsed or refractory multiple myeloma. Blood Adv. 2017;1(23):2105-2114

29. Pomeroy EJ, Hunzeker IT, Kluesner MG, et al. A genetically engineered primary human natural killer cell platform for cancer immunotherapy. Mol Ther. 2019;28(1):5263

30. Kararoudi MN, Dolatshad H, Trikha P, et al Generation of knock-out primary and expanded human NK cells using Cas9 ribonucleoproteins. J Vis Exp. 2018;2018 (136):58237

31. Taussig DC, Miraki-Moud F, Anjos-Afonso F, et al. Anti-CD38 antibody-mediated clearance of human repopulating cells masks the heterogeneity of leukemia-initiating cells. Blood. 2008;112(3):568-575

32. Szegezdi E, Reis CR, Sloot AM van der, et al. Targeting AML through DR4 with a novel variant of rhTRAIL. J Cell Mol Med. 2011:15(10):2216-2231.

33. Gao Z, Tong C, Wang Y, Chen D, Wu Z, Han W. Blocking CD38-driven fratricide among $T$ cells enables effective antitumor activity by CD38-specific chimeric antigen receptor $\mathrm{T}$ cells. J Genet Genomics. 2019:46(8):367-377.

34. Cichocki F, Woan K, Wu C-Y, et al. NK cells lacking CD38 are resistant to oxidative stress-induced death. Blood. 2019;134(Supp 1):3215-3215 